\title{
Saberes, expertos e instituciones vinculadas al universo del movimiento. El caso del VII Congreso Panamericano de Educación Física, Dominicana 1980
}

\author{
Knowledge, experts and institutions linked to the movement universe. The case \\ of the VII Pan American Congress of Physical Education, Dominican 1980
}

Pablo Scharagrodsky ${ }^{1}$ ORCID: 0000-0001-6305-2017

Recibido: 8/04/2021 • Aprobado: 27/04/2021

\begin{abstract}
Cómo citar: Scharagrodsky, P. (2021). Saberes, expertos e instituciones vinculadas al universo del movimiento. El caso del VII Congreso Panamericano de Educación Física, Dominicana 1980. Ciencia y Educación, 5(3), 145-160. Doi: https://doi.org/10.22206/ cyed.2021.v5i3.pp145-160
\end{abstract}

\section{Resumen}

El siguiente trabajo analiza, a partir de una historia social, política y cultural de la educación, en diálogo con la historia de la educación física como disciplina escolar, el VII Congreso Panamericano de Educación Física realizado en Santo Domingo en 1980. Indaga al evento como un lugar de sociabilidad, transmisión de saberes, construcción de lazos personales e institucionales y consolidación de redes transnacionales. Teniendo esto en cuenta, el objetivo central es identificar el tipo de circulación de saberes y los significados transmitidos en el congreso panamericano, así como los circuitos transnacionales y los grupos de expertos presentes, reconocidos y legitimados por el resto de la especialidad. Entre las conclusiones, se identifica al congreso como un espacio consolidado de intercambio entre expertos de diferentes países e instituciones con un fuerte apoyo estatal, el predominio de ciertos enfoques pedagógicos (tecnicismo educativo) y el afianzamiento de redes transnacionales vinculadas a la educación física y los deportes.

Palabras clave: congreso; educación física; circulación de saberes; especialistas; redes transnacionales.

\begin{abstract}
Based on a social, political and cultural history of education and in dialogue with the history of physical education as a school discipline, the following work analyzes the VII Pan-American Congress of Physical Education held in Santo Domingo in 1980. It investigates the event as a place of sociability, transmission of knowledge, construction of personal and institutional ties and consolidation of transnational networks. Taking this into account, the main objective is to identify the type of knowledge circulation and the meanings transmitted in the Pan-American congress, as well as the transnational circuits and the groups of experts, recognized and legitimized by the rest of the specialty. Among the conclusions, the congress is identified as a consolidated space for exchange between experts from different countries and institutions with strong state support, the predominance of certain pedagogical approaches (educational technicality) and the consolidation of transnational networks linked to physical education and sports.
\end{abstract}

Keywords: Congress; physical education; knowledge circulation; specialists; transnational networks.

a Universidad Nacional de Quilmes, Argentina. Universidad Nacional de La Plata, Argentina. Correo-e: pas@unq.edu.ar 
Saberes, expertos e instituciones vinculadas al universo del movimiento.

El caso del VII Congreso Panamericano de Educación Física, Dominicana 1980

\section{Introducción}

En el año 1980 se realizó el VII Congreso Panamericano de Educación Física en Santo Domingo. El evento realizado en la capital de la República Dominicana - una de las ciudades más antiguas del Caribefue el resultado de casi cuarenta años de intercambios, reflexiones, negociaciones, lazos y vinculaciones entre un sector significativo de la comunidad de especialistas en Educación Física, medicina deportiva y recreación de la mayoría de los países americanos.

Desde el ańo 1943, los eventos panamericanos de Educación Física se convirtieron en espacios de debate y discusión sobre las finalidades, las políticas y los medios que debían adoptar los sistemas educativos y las instituciones deportivas en los estados nacionales y las sociedades latinoamericanas a la hora de educar los cuerpos en movimiento, gestionar determinadas políticas deportivas, promocionar actitudes vinculadas con la vida 'sana' o demandar mayores recursos materiales y económicos para materializar las múltiples intervenciones definidas por ese grupo ocupacional.

Estos espacios de sociabilidad en los que circularon ideas, personas, saberes, proyectos y políticas tuvieron su primer acto 'fundacional' con el Primer Congreso Panamericano de Educación Física, realizado en Río de Janeiro en 1943. Tres años después, en 1946, la organización del segundo estuvo auspiciada por México. El tercero se concretó en Montevideo, en 1950; el cuarto fue organizado en Bogotá, en 1965; el quinto se realizó en Buenos Aires, en 1970; y el Sexto Congreso Panamericano de Educación Física se llevó a cabo en Venezuela, en 1976.

La participación de representantes y especialistas dominicanos estuvo ausente en los primeros eventos, incorporándose algún delegado o especialista en los eventos posteriores. Por ejemplo, en el primero República Dominicana no tuvo representantes o participantes ( $\mathrm{O}$ que foi o I congresso panamericano de educação física, 1944, pp. 20-30). Lo mismo sucedió en el Segundo, Tercero y Cuarto Congreso Panamericano de Educación Física, donde no hubo participa- ción de expertos dominicanos en el tema (Memoria oficial del II Congreso Panamericano de Educación Física, 1946, pp. 343-353; Informe del III Congreso Panamericano de Educación Física, 1950; Documentos de Trabajo, Memorias del IV Congreso Panamericano de Educación Física, 1965). Recién en el Quinto Congreso Panamericano de Educación Física, realizado en Argentina, con más de mil trescientos congresistas pertenecientes a trece países de América, por primera vez hubo un representante de la República Dominicana: Rafael M. Ortiz Celado (Memoria del V Congreso Panamericano de Educación Física, 1971, pp. 8-135). En el Sexto Congreso Panamericano de Educación Física, realizado en Venezuela, con la participación de dieciséis países, nuevamente la participación dominicana dijo presente con un especialista: Tomás Ramírez (VI Congreso Panamericano de Educación Física y II Gimnasiada Americana, Tomo I, 1976, p. 3).

Aunque en el balance general la participación de la República Dominicana en esos eventos fue reducida en comparación con el resto de los países, luego de distintos debates y diferentes propuestas, se acordó que el VII Congreso Panamericano de Educación Física se realizara en Santo Domingo.

Teniendo en cuenta el evento, el siguiente trabajo analiza las propuestas pedagógicas desarrolladas en el VII Congreso Panamericano de Educación Física realizado en la capital de la República Dominicana. Para esto, indaga al espacio académico internacional como un lugar de sociabilidad, transmisión de saberes, construcción de lazos personales e institucionales y definición epistémica de criterios para orientar a la disciplina educación física, los deportes y el currículo escolar en los diferentes sistemas educativos latinoamericanos.

\section{Metodología}

En función al universo indagado (el congreso panamericano realizado en Santo Domingo en 1980), la estrategia metodológica se nutrió de un enfoque sociohistórico, con énfasis en la historia social, política y cultural de la educación (Arata \& Pineau, 2019), 
en diálogo con la historia de la educación física como disciplina escolar y de los deportes (Goodson, 2003; Melo \& Fortes, 2010; Torres \& Scharagrodsky, 2019; Galak, 2019; Quitzau \& Moraes e Silva, 2020).

Esto significa pensar al congreso como un espacio material y simbólico de acuerdos y disputas en el que distintos agentes, actores, instituciones y grupos sociales pusieron en circulación, distribución, transmisión y producción un conjunto complejo y heterogéneo de significados vinculados con la educación de los cuerpos en movimiento, que no solo habilitaron y potenciaron ciertos saberes, prácticas o enfoques pedagógicos, sino que, a la vez, produjeron el fenómeno y los objetos a los que hicieron alusión (Scharagrodsky, 2014). Vale decir, los congresos representaron un aspecto de la compleja trama educativa y social, pero también tuvieron un carácter performativo (Butler, 2019), es decir, de construcción de la realidad educativa vinculada con la educación física y los deportes. Aquello que fue enunciado por ciertos referentes o expertos terminó, de alguna manera, siendo la Educación Física.

Teniendo en cuenta lo antes planteado, el objetivo central del trabajo es identificar la circulación de saberes y los significados transmitidos en el congreso panamericano, así como los grupos de expertos presentes, reconocidos y legitimados por el resto de la especialidad. Asimismo, es la intención identificar las redes personales e institucionales que se posicionaron en ese espacio. Entre las interrogantes planteadas es posible mencionar las siguientes: ¿cuáles fueron los tópicos que se debatieron en el evento? ¡cuáles enfoques pedagógico-didácticos prevalecieron?, ¿cuáles fueron las mayores discordias o tensiones vinculadas a las temáticas abordadas? ¿quiénes fueron los expertos y las instituciones con el derecho autorizado a enunciar las verdades sobre la educación física y los deportes?

Para llevar a cabo el análisis hermenéutico que articula las lógicas internas del congreso con el contexto social, político y educativo, se ha focalizado la atención interpretativa en diversas fuentes documentales, entre las que se destacan las memorias e informes del congreso, la prensa escrita y el tipo de visibilidad fabri- cada, las exposiciones centrales de los expertos, las ponencias generales y las memorias vinculadas a otros congresos panamericanos.

Por la naturaleza del problema de investigación, se aplicó una metodología cualitativa de carácter exploratorio. La metodología cualitativa es consistente con el interés de nuestro trabajo en acceder a una comprensión más compleja y profunda del fenómeno de estudio, que nos coloque ante la posibilidad de contribuir a sumar conocimiento interpretativo y elaborar hipótesis sustantivas. Nuestra hipótesis general sugiere que el Séptimo Congreso Panamericano de Educación Física, realizado en Santo Domingo a principios de los años ochenta, afianzó un espacio de sociabilidad e intercambio entre agentes de diferentes países e instituciones con un fuerte apoyo estatal, el cual consensuó ciertos temas (formas de elaborar el currículo, de definir a la educación física y sus objetivos, de proyectar mejores dispositivos de formación o de pensar al deporte escolar), bajo ciertos enfoques (fundamentalmente la perspectiva tecnicista de la educación) y, al mismo tiempo, legitimó a ciertos expertos de la especialidad como los máximos referentes y a determinadas redes trasnacionales como los circuitos más significativos donde reclamar y definir políticas y acciones vinculadas a la educación física, los deportes escolares y la recreación.

\section{Resultados}

\section{El evento panamericano: jerarquización de temas y significados puestos en circulación}

El Séptimo Congreso Panamericano de Educación Física fue organizado y apoyado política y económicamente por diversas estructuras estatales y distintos organismos privados, entre ellos se destacaron la Secretaría de Estado de Educación, Bellas Artes y Cultos de la República Dominicana, la Secretaría de Deportes, Educación Física y Recreación, la Universidad Autónoma de Santo Domingo, el Club Deportivo y Cultural San Lázaro y el Gulf and Western Americas Corporation.

En términos macropolíticos, el congreso se realizó en un contexto latinoamericano heterogéneo, 
pero, al mismo tiempo, fuertemente atravesado por procesos parciales de modernización cuyo lado más violento e injusto estuvo vinculado -en las décadas de los sesenta y setenta- con la dependencia, el subdesarrollo, la desigualdad social, la lenta declinación del keynesianismo, la decadencia de las teorías del desarrollo, los procesos de militarización traducidos en golpes cívico-militares y la violencia política (Arnove, 2006; Serrano, 2010). En el campo específicamente educativo:

[...] las décadas de sesenta y setenta (son) un punto de inflexión de los sistemas educativos de la región, que bajo la consigna modernizadora introducirá cambios en las modalidades de intervención estatal a través de la planificación, difundirá nuevas corrientes del pensamiento educativo y marcará el surgimiento de nuevos profesionales, que en conjunto complejizarán las formas de regulación y gobierno del sistema. [...] Como parte de este mismo proceso, la difusión de las teorías de capital humano y de formación de recursos humanos en el campo educativo no solo darían cuenta del valor económico de la educación y la importancia estratégica de mejorar las calificaciones de la fuerza de trabajo sino también aportarían un conjunto de metodologías que conformarán el llamado 'planeamiento educativo'. (Suasnábar, 2017, pp. 116-117)

Este último ítem atravesó buena parte de las discusiones en las distintas ponencias centrales. El planeamiento educativo, el diseño curricular por etapas, la definición de objetivos operacionales y direccionales o la formación de recursos humanos en el área para lograr mayor eficiencia en la enseñanza fueron aspectos altamente ponderados para mejorar a la educación física y al profesor de esa disciplina. Fue en este particular contexto que se realizó en noviembre de 1980 el evento internacional.

La ceremonia de apertura del congreso en el Hotel Plaza Dominicana tuvo la presencia de reconocidas autoridades estatales de la República Dominicana; por ejemplo, el Secretario de Estado de Educación, Ingeniero Pedro Porrello Reynoso. Como presidente del Comité Organizador del evento internacional pronunció un discurso que combinó la concreción de supuestos ideales morales necesarios para la vida cotidiana moderna, junto con definiciones generales sobre la disciplina.

Además de autoridades nacionales, como "el general de brigada Luciano Díaz y Díaz, los subsecretarios Rafael Valdez Hilario y el coronel Marcos A. Jiménez, Andrés Reyes, entre otros" (Rodríguez, 17 de noviembre de 1980), la apertura del congreso panamericano tuvo la presencia de reconocidos y distinguidos especialistas nacionales e internacionales vinculados con la Educación Física y el universo deportivo. Entre ellos se destacaron, como en los últimas tres décadas, el famoso profesor argentino Enrique Carlos Romero Brest (Saraví Rivière, 2014), que estuvo en calidad de presidente del estratégico Comité de Planificación de los Congresos Panamericanos de Educación Física, y el profesor y director general de Educación Física de la República Dominicana José Sánchez Pérez, quien fue nombrado presidente del congreso y vicepresidente del comité organizador.

Entre los 400 asistentes al evento internacional hubo delegaciones de diferentes países: "Chile, Brasil, Costa Rica, Cuba, Curazao, España, Alemania, Guyana Británica, Haití (...), Igualmente de Honduras, Inglaterra, Jamaica, Panamá, Uruguay, Perú, Paraguay, Puerto Rico, Venezuela, Colombia, Francia, Bélgica, México y República Dominicana” (Rodríguez, 15 de noviembre de 1980), en el marco de procesos de intercambio y diálogos transnacionales sobre la cultura física y la educación física cuya historia entre Europa y América se remontaba a finales del siglo XIX o principios del XX (Gleyse \& Scharagrodsky, 2013/2; Linhales, Rodrigues Puchta \& Rosa, 2019). Sin embargo, este proceso se consolidó en la segunda mitad del siglo XX a través de convenios y acuerdos formales entre diversos países latinoamericanos y otros países, entre los que sobresalieron muy especialmente Alemania, pero también España, Francia y Estados Unidos para Colombia, Argentina, Chile, México, etc. y la URSS -y otros países de la órbita soviética- para el caso de Cuba (Eusse, Almeida \& Bracht, 2019; Levoratti, 2020). 
Como solía suceder, la amplia mayoría de los asistentes al evento provenía de países americanos, aunque a partir de la década de los ańos setenta -lentamente y en un número reducido- se incorporaron delegados y expositores de países europeos, especialmente aquellos vinculados con la producción de saberes, objetos, enfoques didácticos y propuestas pedagógicas relacionados con la educación física moderna (Kirk, 2010; Scharagrodsky, 2011). En general, la mayoría de los congresistas estuvo relacionada al mundo de la docencia (profesores de educación física, maestros, etc.) o al amplio universo deportivo (entrenadores, exdeportistas, dirigentes deportivos, idóneos, etc.), pero también hubo otras profesiones interesadas en el heterogéneo universo de la cultura física como los médicos deportólogos, los kinesiólogos, los periodistas deportivos y los arquitectos interesados en las edificaciones deportivas.

Como era costumbre en este tipo de congresos, se realizaron performances corporales vinculadas con la educación física y el deporte: “(...) un grupo de niños realizó una demostración, en representación de la niñez dominicana”. “(...) Además, el grupo de bailes folklóricos de la Secretaría de Turismo realizó una representación" (Rodríguez, 17 de noviembre de 1980) (Figuras 1 y 2). La visibilidad de la cultura dominicana y su especificidad reforzó, ante la atenta mirada de los delegados extranjeros, las particularidades de las expresiones corporales del país anfitrión, exhibiendo y ponderando ciertos rasgos identitarios de la 'dominicanidad' pero, al mismo tiempo, los combinó con una narrativa latinoamericanista. Este tipo de representaciones también sumó y valoró la música nacional y extranjera "(..) con la presentación del Coro Nacional de Bellas Artes (...) en los salones del Hotel Plaza Dominicana” (Rodríguez, 19 de noviembre de 1980) y también en las Ruinas de San Francisco (Figura 3). Asimismo, el evento seleccionó a uno de los símbolos teatrales de la identidad del país organizador: el famoso Teatro Rodante Dominicano, el cual se presentó ante un numeroso público en la Universidad Nacional Pedro Henríquez Ureña.

\section{Figura 1}

Inauguración del VII Congreso Panamericano de Educación Física

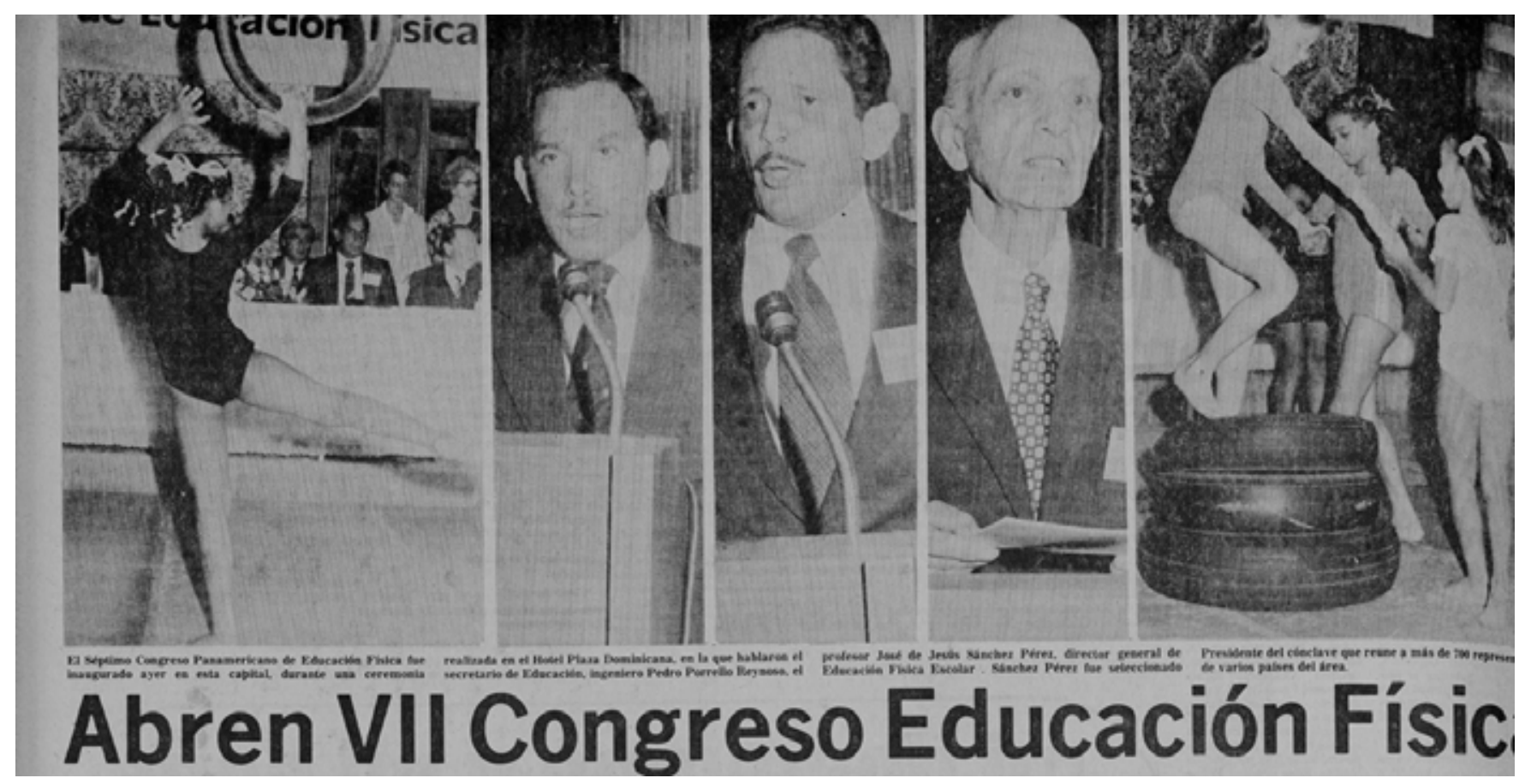

Nota: la fuente de la figura es Rodríguez, R. (17 de noviembre de 1980), Abren VII Congreso de Educación Física. El Caribe. 
Saberes, expertos e instituciones vinculadas al universo del movimiento.

El caso del VII Congreso Panamericano de Educación Física, Dominicana 1980

\section{Figura 2}

Participación de grupos de bailes folklóricos durante la inauguración del congreso

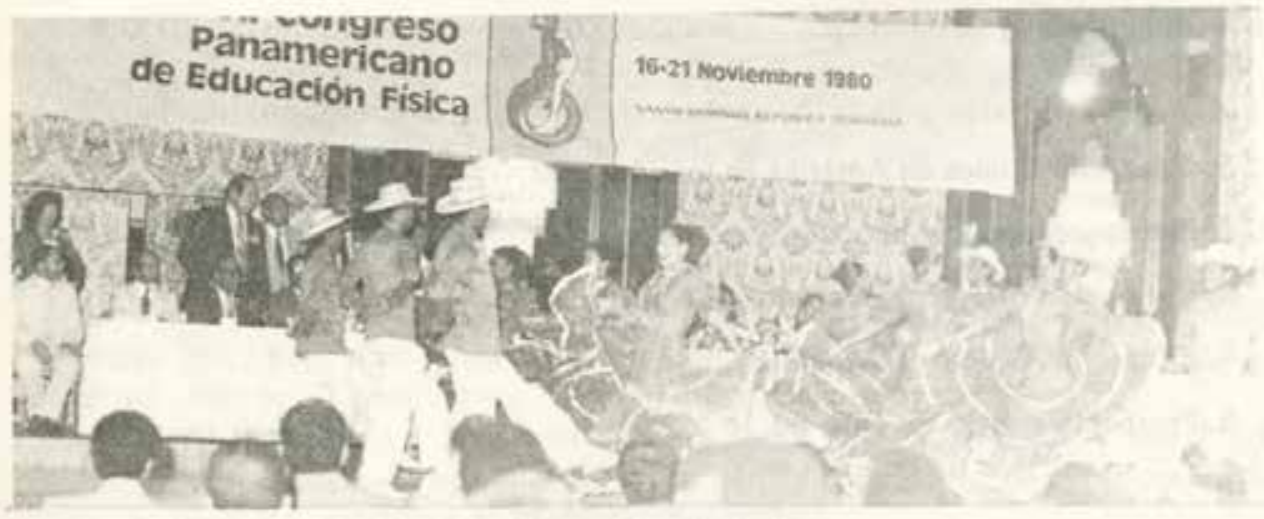

3.-Aspecto de la participación del grupo de bailes folklóricos de la Secretaría de Estado de turismo, actuación que fue aplaudida por los presentes.

Nota: la fuente de la figura es la Memoria oficial del VII Congreso Panamericano de Educación Física, 1980, p. 9.

\section{Figura 3}

Presentación del Coro Nacional en las ruinas de San Francisco

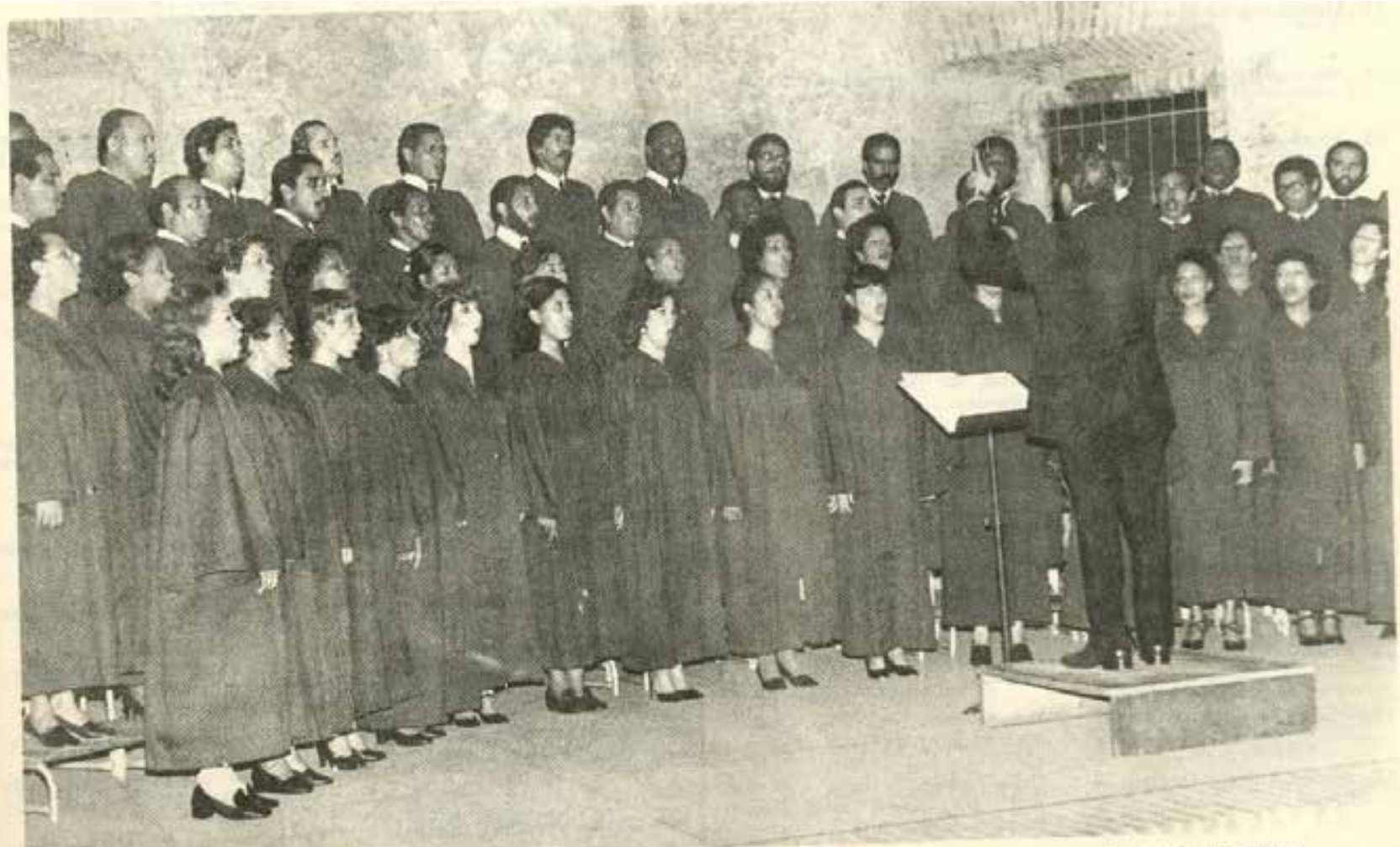

Una de las actividades cuiturales del Congreso: la presenta- ción del Coro Nacional, acto que tuvo lugar en las Ruinas de San Francisco.

Nota: la fuente de la figura es la Memoria oficial del VII Congreso Panamericano de Educación Física, 1980, p. 97. 
Pero ¿qué tipo de temas se pusieron en discusión? Las secciones del congreso, los tópicos debatidos y las discusiones generadas nos brindan indicios sobre cómo fue conceptualizada la disciplina, cuáles enfoques pedagógicos fueron priorizados, cuáles fueron las funciones educativas y políticas asignadas, las discordias producidas y qué tipo de reclamos generales se ensayaron y a quiénes estaban dirigidos en ese evento (Scharagrodsky, 2020).

El VII Congreso Panamericano de Educación Física presentó como tema central de discusión "La Educación Física en América en los años '80" (Rodríguez, 18 de noviembre de 1980). Ese universo se subdividió en cinco temas: "Educación Física en América en los años '80"; "planes y programas actuales"; "formación de recursos humanos necesarios"; "educación física y motricidad" y "prospectivas para los años '80". La dinámica del evento incluyó exposiciones centrales con invitados especiales sobre los cinco temas mencionados, así como espacios de debate sobre esos tópicos y ponencias o presentaciones más generales.

El tema vinculado con la educación física en América en los '80 fue desarrollado por el profesor argentino Mario López, quien ya había estado presente en los congresos de la década de los setenta. Su exposición central retomó el lugar de la educación física en el proceso educativo moderno proponiendo "un modelo de hombre creativo". Desarrolló su exposición a partir de nociones específicas como maduración, crecimiento y aprendizaje (Memoria oficial del VII Congreso Panamericano de Educación Física, 1980, pp. 11-12). Su propuesta, con matices, estuvo fuertemente atravesada por una concepción tecnocrática de la educación que aún estaba muy presente en los años sesenta y setenta en muchos países de América latina. De hecho, buena parte de la presentación focalizó la atención en la descripción y explicación de los objetivos direccionales ("son formulaciones enunciadas en términos de conductas") y de los operacionales ("son los objetivos concretos de aprendizaje en términos de conductas observables"), con el fin de desarrollar la formación física básica, la educación del movimiento, el rendimiento motor, la creatividad y la formación física técnica. Todas ellas dimensiones estructurales de la disciplina, ya presentes en los años setenta, en las currículas de muchos países del continente americano.

Retomando y citando a "Mager, Gagne o Bloom" (Memoria oficial del VII Congreso Panamericano de Educación Física, 1980, pp. 13-14), el paradigma formulado por Mario López se centró, muy especialmente, en cuestiones de organización y desarrollo de la práctica con un sentido vinculado a medios y fines racionales. En su exposición hubo una obsesión que se condensó en la siguiente afirmación: todo comportamiento útil del alumno debe ser medible, empíricamente registrable y claramente observable. Su insistencia en que los objetivos debían ser formulados en términos de comportamientos explícitos, verificables, mensurables y cuantificables lo ubicó más cerca de la tradición que valorizó más el cómo de la educación física que el por qué y el para qué (Díaz Barriga, 1996). Esta orientación se radicalizó en algunos países y actores educativos en los años sesenta y setenta, con la revitalización de una tendencia fuertemente tecnicista en la educación estadounidense, representada sobre todo por un libro de Robert Mager, llamado Análisis de objetivos, muy influyente en muchos países de sudamérica (Silva, 2001).

La segunda exposición central del evento problematizó la cuestión de los planes y programas y la desarrolló el profesor Emilio Guerra, en representación del Instituto Pedagógico de Caracas, Venezuela. Hizo un balance de la educación y el deporte en varios países de América, ponderando a EE. UU., Canadá y Cuba, aunque criticó el mundo subdesarrollado latinoamericano instando a "romper las cadenas de inferioridad y minusvalía y atender el reto del desarrollo" (Memoria oficial del VII Congreso Panamericano de Educación Física, 1980, p. 43). Su enfoque fue distinto en términos conceptuales al de Mario López, ya que insistió en que "(...) el desarrollo deportivo de un país se fundamenta en la Educación Física escolar (...) (Memoria oficial del VII Congreso Panamericano de Educación Física, 1980, p. 36), generando con esto un cierto sentido de dependencia de la disciplina educativa ante la omnipresente lógica deportiva. Asimismo, 
retomó la importancia de los programas de entrenamiento físico (resistencia aeróbica, anaeróbica, flexibilidad, fuerza muscular, etc.) y de las evaluaciones previas, así como de programas de educación física adaptados a niños "física o moralmente impedidos" (Memoria oficial del VII Congreso Panamericano de Educación Física, 1980, p. 40) y resaltó la importancia de la formación de un buen profesor de educación física. A nivel curricular, propuso tener en cuenta cuatro aspectos básicos: deporte para la vida, filosofía del humanismo, alternativas para la formación profesional y propósitos y procesos curriculares. Sus críticas a la tradición disciplinar estuvieron presentes:

[...] las clases de Educación Física tradicionalmente se caracterizaban por métodos autoritarios, organización formal, dirección rígida por parte del profesor, muchos reglamentos, códigos de conducta y el no reconocimiento de las diferencias individuales (proponiendo) mayor énfasis a la libertad, el valor, a la dignidad o integridad del individuo. (Memoria oficial del VII Congreso Panamericano de Educación Física, 1980, p. 48)

Estos conceptos estuvieron matizados con algunas categorías tecnocráticas provenientes de producciones como, por ejemplo, las de Hilda Taba, quien fue citada como una referencia importante en su trabajo (Memoria oficial del VII Congreso Panamericano de Educación Física, 1980, p. 51).

Por otra parte, la exposición vinculada con la formación de recursos humanos fue desarrollada por la profesora colombiana Martha Moncada de Rojas, quien estuvo relacionada con Coldeportes del Ministerio de Educación de Colombia y también con algunas universidades de ese país. La presentación planteó "aspectos generales del currículo, el cambio y el futuro (...)”. Su posición partió "del principio de un currículo centrado en el alumno (...)”. No obstante, la influencia tecnocrática siguió estando presente retomando la tradición planteada por algunas referencias curriculares como las de Hilda Taba y la forma de pensar la organización curricular de la educación física centrada en la importancia del diseño racional e integrado, de acuerdo a ciertas dimensiones:
Fundamentos del curriculum: A. el educando, B. la materia del contenido, C. la cultura, D. la sociedad, E. principios y leyes del aprendizaje, F. filosofía del sistema educativo y G. función de la escuela. [...] Componentes del currículo: objetivos, contenido temático, metodología, servicios educativos, administración, recursos didácticos y evaluación. (Memoria oficial del VII Congreso Panamericano de Educación Física, 1980, pp. 55-57; Moncada de Rojas, 1981)

Por último, hubo dos importantes exposiciones: una vinculada con la educación física y la motricidad y la otra con las prospectivas para los años ochenta. La primera fue desarrollada por el reconocido profesor norteamericano Robert Singer. Se centró "en el aprendizaje de las habilidades psicomotoras" como uno de los objetivos centrales de la educación física. Su propuesta retomó analogías corporales entre el hombre y el animal y propuso, a partir de algunas categorías provenientes de la biomedicina, la mejor forma de desarrollar habilidades motoras simples y complejas. Asimismo, articuló su desarrollo con conceptos y nociones provenientes del campo psicológico, como la percepción, la motivación, el estado psicológico, la gratificación a través del elogio o el procesamiento de información. Su propuesta pedagógica combinó su doble formación académica vinculada con el aprendizaje motor y la psicología del deporte. Concluyó que "los programas de educación física necesitan ser estructurados más sistemáticamente, para que muchos estudiantes puedan lograr mejor su potencial" (Memoria oficial del VII Congreso Panamericano de Educación Física, 1980, pp. 60-62). La insistencia en la planificación fue un aspecto central de su presentación.

La otra exposición central del evento -quinta y última- fue desarrollada por el arquitecto venezolano Carlos Vera Guardia, figura clave en el campo de la educación física latinoamericana por las próximas dos décadas, versó sobre la educación física actual y la educación física del futuro. Su propuesta, retomando las conclusiones de la UNESCO, situó a la educación física como parte de "la educación integral" a partir de la "gimnasia, los deportes y la recreación" 
(Memoria oficial del VII Congreso Panamericano de Educación Física, 1980, pp. 67-68). Planteó el trabajo de actividades físicas utilizando el criterio de las edades ( 0 a 6 años, 7 a 12 años, 13 a 18 años, etc.). Entre las conclusiones mencionó que:

La mayor cantidad de actividades de la educación física en las diversas edades del hombre, se dan en la edad de 7 a 12 años en forma de prácticasa obligatorias en las que las actividades gimnásticas y deportivas tienen prioridad; pero aún en ese grupo el porcentaje de población es demasiado bajo y las actividades son muy pobres consideradas las reales necesidades del niño. (Memoria oficial del VII Congreso Panamericano de Educación Física, 1980, p. 70)

Según el autor, las razones de esta situación estuvieron relacionadas con la falta de reconocimiento a la especialidad, la carencia de políticas gimnásticas, deportivas y recreativas y la escasez de recursos humanos capacitados. Entre los aportes de la educación física del futuro acudió a los trabajos provenientes de la psicomotricidad de Le Boulch y Vayer (Memoria oficial del VII Congreso Panamericano de Educación Física, 1980, pp. 74-75) y sugirió ampliar el rango de trabajo más allá de la población vinculada al sistema educativo formal.

Muchos de estos conceptos fueron retomados en los espacios de discusión y expuestos en algunas ponencias como la de Alberto Abrego, quien expuso sobre planes y programas; Alberto Calderón y José Ospina, quienes problematizaron la evaluación de programas de educación física para la formación docente; Jacinto Targa, que abordó la psicomotricidad como uno de los caminos para los problemas de la educación física; o Raúl Horacio Gómez que avanzó sobre la fundamentación vinculada a la iniciación deportiva.

Entre las discusiones y tensiones producidas en el congreso, además de algunas críticas a la tradición autoritaria en la enseñanza de la disciplina, la necesidad de mejorar la formación profesional, ampliar racionalmente la planificación y aumentar los recursos económicos, apareció -como en los anteriores congresos- el tema del deporte y su compleja y contradictoria relación con la educación física:
Un tema controversial durante el congreso fue el referente a la relación del deporte y la educación física. El congreso aclaró que la estructura educativa encuentra en el deporte un medio importante para formar valores y observó la necesidad de que se evite las posibles influencias deformantes del deporte de alto nivel competitivo (Rodríguez, 22 de noviembre de 1980). [...] la educación física no ha llegado a su máximo desarrollo debido a que generalmente se la confunde con la práctica del deporte. (Rodríguez, 21 de noviembre de 1980)

De hecho, parte de la prensa presente en el evento señaló que los "expositores definieron a la educación física como parte del proceso integral del individuo y en seguida se preguntaron: si la temprana especialización deportiva puede significar en millones de niños de nuestros países (...) curriculums con tendencias hacia la enseñanza diversificada?" (Rodríguez, 20 de noviembre de 1980). Esto se visibilizó en las conclusiones del congreso donde se mencionó “(...) la disparidad de criterios en cuanto a la edad en que ha de iniciarse la actividad deportiva" (Memoria oficial del VII Congreso Panamericano de Educación Física, 1980, p. 84).

En este contexto, de acuerdos y disputas de sentidos sobre la educación física, la enseñanza, el aprendizaje, la iniciación deportiva escolar, etc., entre las conclusiones generales de los y las congresistas se insistió plantear claramente "los objetivos direccionales desde un enfoque psico-motor, estableciéndose la necesidad de definir los del área socio-afectiva y cognoscitiva (...), teniendo en cuenta los sexos" y las etapas etarias mencionadas, así como los objetivos operacionales "los que orientarán la selección y ordenamiento de contenidos, la creación de actividades, la selección de recursos (...)". Con respecto al deporte, se le mencionó como medio educativo, pero "procurando evitar las posibles influencias deformantes del deporte de alto nivel competitivo". Además, se planteó la necesidad de ampliar los recursos disponibles para mejorar las políticas deportivas y recreativas de parte del Estado y de las instituciones privadas, más allá del ámbito educativo formal y, también, 
mejorar la formación profesional, vincularla "con la universidad" y "elaborar planes y programas aprovechando al máximo los recursos existentes del más alto beneficio al menor costo posible" (Memoria oficial del VII Congreso Panamericano de Educación Física, 1980, pp. 83-87).

\section{El evento: el tránsito de los expertos y las institu- ciones participantes}

El congreso no solo puso en circulación un conjunto de saberes, problemas y enfoques que fueron delineando una determinada forma de conceptualizar a la educación física, al currículum, a los objetivos, a la enseñanza, al aprendizaje, a los deportes, al profesor de educación, o a un tipo de relación pedagógica, sino que también visibilizó y, en parte, legitimó a un grupo de expertos, quienes se convirtieron en los verdaderos productores de sentidos de y sobre la especialidad, así como en definidores de ciertas políticas llevadas a cabo en cada uno de sus países americanos.

Lentamente se fueron constituyendo grupos de expertos en el campo de la cultura física, en general, y de la educación física, en particular, los cuales, amparados en ciertas instituciones (Direcciones Generales de Educación Física o de deportes, instituciones de formación docente, universidades, clubes deportivos, organismos sanitarios, instituciones militares, etc.), se convirtieron en los legítimos productores de los sentidos específicos sobre la Educación Física y sus diferentes finalidades. Aquello que enunciaron como Educación Física, deporte, recreación o currículum se convirtió, de hecho, en la 'auténtica' Educación Física, el deporte, la recreación o el currículum.

En este contexto, entre los referentes o expertos dominicanos de la especialidad, además del profesor y director general de Educación Física, José Sánchez Pérez, estuvieron presentes el profesor Ramón Aybar, Onaney Girón de Herrand, Abelardo Díaz, Rolando Miranda, Francisco Pelletier, Danilo Aquino, Tomás Ramírez, César Padrón y el Dr. Williams Coss. Algunos de ellos con una trayectoria de años en la especialidad y otros con importantes vínculos en el mundo educativo o deportivo dominicano.
Entre los expertos extranjeros se destacaron, además del ya mencionado Enrique Carlos Romero Brest, figuras de 'talla' internacional como "Carlos Vera Guardia, Liselott Diem, Jacinto Targa, Oscar Rendoll Gómez y Joselim Ramos" (Rodríguez, 17 de noviembre de 1980). Asimismo, sobresalieron especialistas de diversos países; por ejemplo, James Breen y Robert Singer, de Estados Unidos; Héctor Barovero y Patricia Marta Cirigliano, de Argentina; Sonia Toro Seda, de Puerto Rico; Mario Argeñal y María del Carmen Cuello de Rivera, de Honduras; Juan Pablo Repetto, de Chile; Jaime Muñoz Campusano, de Ecuador; Alberto Abrego, de Panamá; Alcibiades Blaires, de Paraguay; Martha Moncada de Rojas y Sabala Cuello, de Colombia; Abraham Ferreiro Toledano y José Jaime Velázquez, de México; Emilio Guerra, Alexander Valero y José Geraldo de Mendoza, de Venezuela; Didier Cerras, de Haití; Mario Araya, de Costa Rica; y María José Lora, de Perú. Varios de ellos con participaciones en congresos anteriores y con destacadas producciones sobre el saber experto socialmente autorizado de la educación física. Muchos estaban vinculados con Direcciones nacionales como la Dirección General de Educación y Deportes de Tegucigalpa, Honduras o la Dirección Nacional de Educación Física, Deportes y Recreación de Argentina; direcciones de educación como el Ministerio de Educación, Básica General de Panamá o ciertos institutos o universidades como el Instituto Pedagógico de Caracas, Venezuela, la Escuela Superior del Profesorado Francisco Morazán de Honduras; o universidades como la de Panamá, la Nacional Autónoma de Honduras, la Nacional de Costa Rica, Universidad del Valle, Colombia, Pontificia Universidad Católica de Puerto Rico, Universidad Estatal de Florida, Estados Unidos, etc.

La visibilidad e importancia de los expertos en la especialidad estuvo dada por quienes tuvieron $-\mathrm{y}$ lograron- el derecho a hablar en el acto de apertura, de clausura, en las exposiciones centrales o aquellos que integraron los espacios de decisión en el congreso. Uno de los espacios más importantes y estratégicos en este tipo de eventos lo constituye el Comité Permanente de los Congresos Panamericanos de Educación Física. Este estuvo integrado por figuras como Enrique 
Carlos Romero Brest (presidente) y los profesores Alberto Langlade, Alberto Gómez Moreno, Oscar Rendoll Gómez, Carl Troester y Carlos Vera Guardia. Varios de ellos con presencia en los panamericanos realizados en 1950 y 1960. La diversidad de nacionalidades (argentina, uruguaya, colombiana, panameña, estadounidense, venezolana, etc.) mostró la injerencia, la influencia o el predominio de ciertos países por encima de otros.
Recordemos que en el acto de clausura "hablaron el profesor José de Jesús Sánchez, presidente del Congreso, el profesor Abraham Ferreiro Toledano, representante de México, el profesor Héctor José Barovero de Argentina, Mario Argeñal de Honduras y el profesor Oscar Rendoll, de Panamá. También el profesor Enrique Romero Brest, de Argentina y Andrés Reyes, subsecretario técnico de la Secretaría de Educación (...)" (Rodríguez, 22 de noviembre de 1980) (ver Figura 4).

\section{Figura 4}

Clausura del Congreso Panamericano de Educación Física

Lัง IN บіАкIU-Viernes 21 de Noviembre de
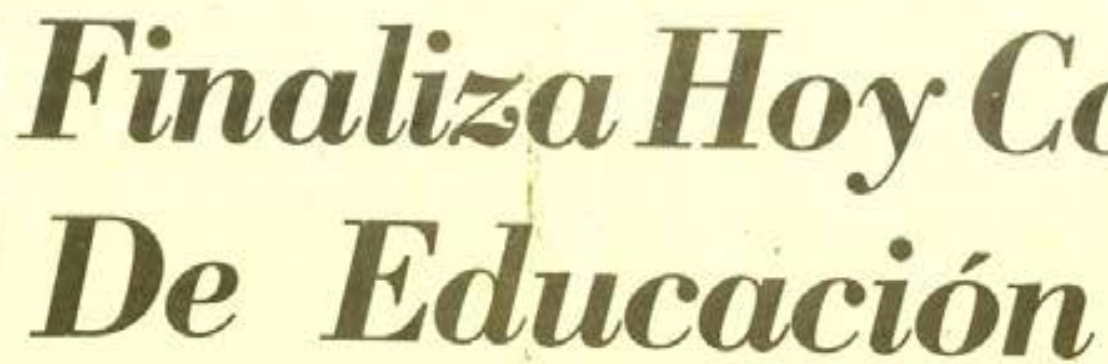

ongreso
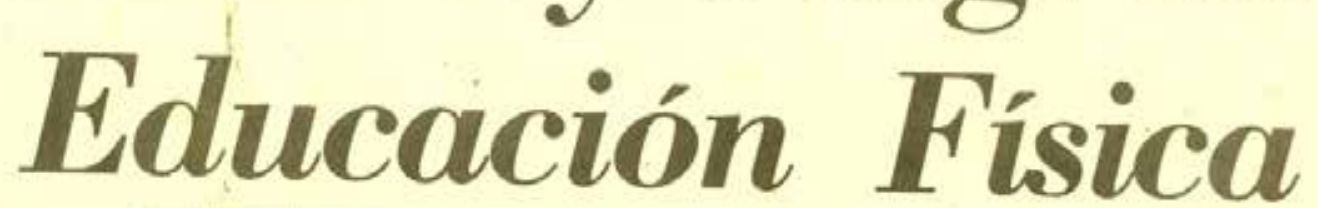

Por Omar Furment

El VII Congreso Pana mericano de Educación Fisica concluye esta noche con la asistencia del presidente de la Republica, Don Antonio Guzmán Fernándes. y su señora esposa, Doถิธ Renée Klang de Guzmán, en el Salón Ambar del Hotel Plaza Dominicana.

El evento se ha desarrollado desde el pasado domingo, que fue cuando se inauguro, con la presencia de más de 300 delegados de diferentes paises del área.

El cónclave es auspi. ciado por la Secretaria de Estado de Educación, Bellas Artes y Cultos.

Por su parte, varios delegados que participan en el congreso han presentado algunos trabajos oficiales, los cuales fueron considerados como de alto contenido cientifico.

Los trabajos fueron presentados por el licenciado Mario Lopez, de Argentina, acerca de la educación física en los afos 80 ; el profesor Emilio Herrera,

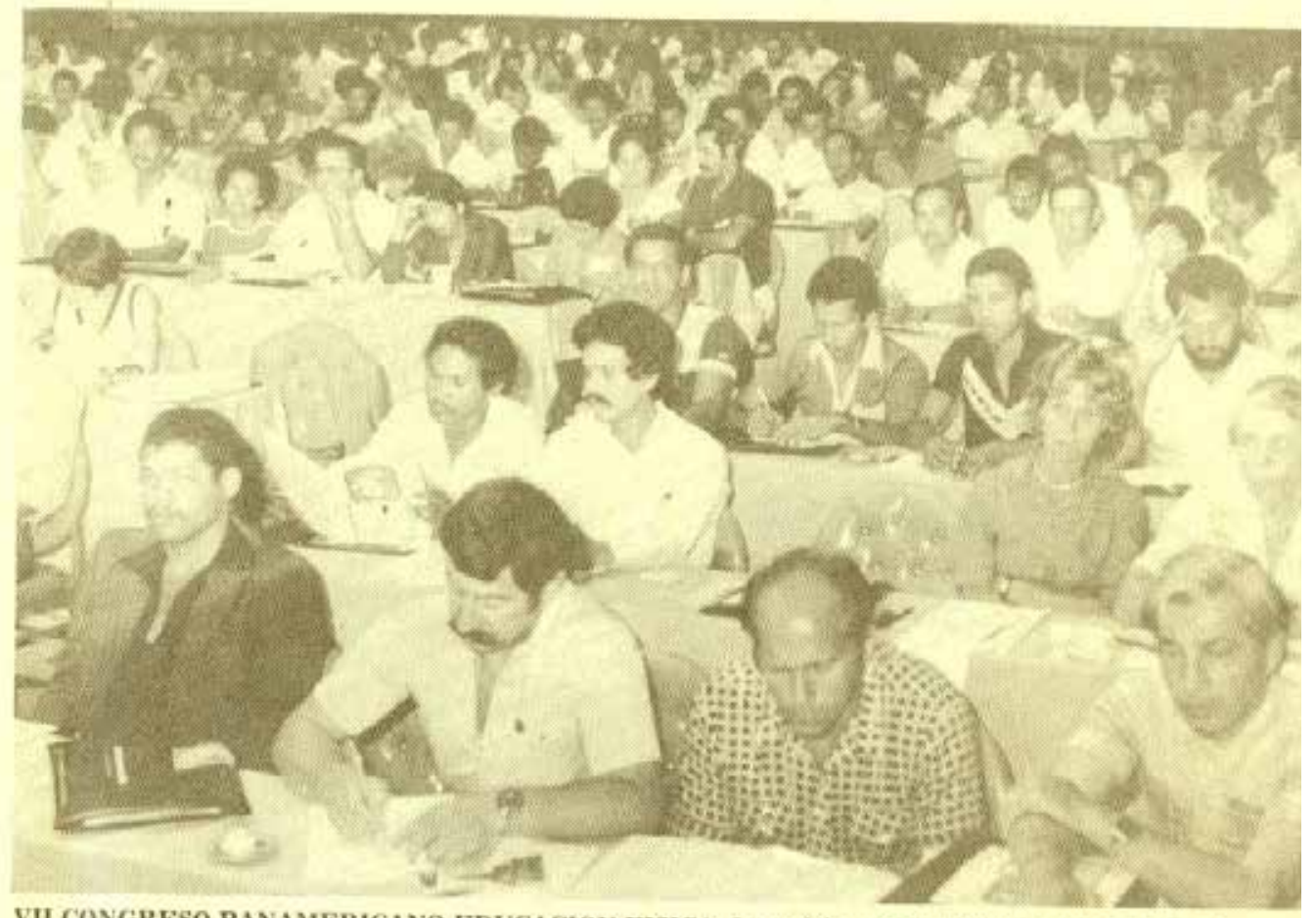

VII CONGRESO PANAMERICANO EDUCACION FISICA. La graficica muestra parte de los delegados que VII Congreso Panamericano de Educación Fisica, que se celebra en el Hotel Plaza Dominicana. El evente
esta noche con la presencia del presidente Antonio Guzmán.

Nota: la fuente de la figura es Furment, O. (21 de noviembre de 1980). Finaliza hoy Congreso de Educación Física. Listín Diario. 
Algunos de los expertos mencionados no solo eran delegados de sus países, sino de organismos internacionales como "Carl Troester de los Estados Unidos (International Council for Health, Physical Education and Recreation), Liselott Diem, presidente de la Federación Internacional de la Educación Física (FIEP) y Jacintho Targa, representante de la FIEP en América” (Rodríguez, 22 de noviembre de 1980). Todos ellos fueron integrantes de instituciones y redes transnacionales de la Educación Física o el mundo deportivo, con importantes recursos materiales y simbólicos y con fuertes vinculaciones con organismos internacionales como la UNESCO, la cual se pronunció en 1978, durante la 20a Conferencia General de la UNESCO acerca de la importancia de la educación física y los deportes en las sociedades modernas, con la famosa Carta Internacional de la Educación Física y el Deporte.

Sin duda, este congreso fue clave en términos de construcción de nuevas alianzas de poder. Lentamente la influencia de los referentes que consolidaron el espacio panamericano en las últimas tres o cuatro décadas fue desplazada o reemplazada por especialistas más jóvenes que disputaron ese lugar de poder y autoridad. Reconocidos expertos como el uruguayo Alberto Langlade, el colombiano Alberto Gómez Moreno o el argentino Enrique Carlos Romero Brest dejaron de gravitar en estos espacios. Por ejemplo, el argentino Enrique Carlos Romero Brest, que fue homenajeado durante el evento, anunció su decisión de apartarse del cargo de presidente del Comité Permanente Panamericano de los Congresos de Educación Física, designándose a partir de ciertas alianzas al profesor y arquitecto Carlos Vera Guardia, quien se convirtió en el responsable de delinear las políticas de los futuros eventos panamericanos durante las próximas dos décadas y consolidar -como nunca antes- redes internacionales con organismos de primera línea.

Por otra parte, a partir de los años ochenta se consolidó un proceso que se había iniciado en los años sesenta y setenta, vinculado con la incorporación de referentes de la especialidad de origen europeo de la mano de redes y alianzas transnacionales que llegaron a la misma UNESCO, consiguiendo no solo visibilidad disciplinar, sino recursos económicos y legitimación política con relación a las intervenciones del profesor de educación física en espacios públicos e instituciones privadas. De hecho, varias ponencias y conferencias centrales citaron la importancia de las distintas organizaciones internacionales existentes vinculadas con la educación física y el deporte a la hora de posicionar y legitimar a este particular grupo ocupacional:

ICHPER (Consejo internacional para la Salud, Educación Física y Recreación), FIEP (Federación Internacional de Educación Física), ICSPE (Consejo Internacional para el Deporte y la Educación Física), COI (Comité Olímpico Internacional), GAIF (Asamblea General de Federaciones Internacionales), GANOL (Asamblea General de los Comités Olímpicos Nacionales), AIESEP (Asociación Internacional de Escuelas Superiores de Educación Física), FISA (Federación Internacional de Deportes Universitarios), FIMS (Federación Internacional de Medicina Preventiva), IASI (Asociación Internacional de Información Deportiva), ICSS (Comité Internacional para la Sociología del Deporte), IFSP (Federación Internacional para la Psicología del Deporte), ICPHS (Comité Intergubernamental para la Educación Física y el Deporte de la UNESCO), ODILAC (Organización Deportiva Internacional de Latinoamérica y del Caribe) (Memoria oficial del VII Congreso Panamericano de Educación Física, 1980, pp. 51-52).

La prensa dominicana ponderó positivamente esta situación: "habrá (...) delegados de reconocidos organismos internacionales especializados en la materia. Entre estos organismos se encuentran la Federación Internacional de Educación Física, el Consejo Internacional para la Educación Física, el Deporte y la Recreación y el reconocimiento de la UNESCO y la OEA" (Rodríguez, 15 de noviembre de 1980), rescatando las múltiples posibilidades de crecimiento de la educación física en la República Dominicana.

Esto también permitió, como en otros congresos, la visibilización de ciertas demandas nacionales solici- 
tando y, en muchos casos, obteniendo recursos materiales y económicos de los organismos internacionales intervinientes. El representante del país anfitrión, José de Jesús Sánchez, señaló: "Nuestra Escuela Nacional (de Educación Física), la cual está siendo remodelada y que en breve será ampliada, estaría en disposición de aceptar cualquier tipo de ayuda de los organismos internacionales con el objeto de alcanzar un alto nivel" (Furment, 21 de noviembre de 1980).

Estos pedidos no solo fueron propiedad del país anfitrión, sino también del resto de los países, especialmente los latinoamericanos. De hecho, entre las conclusiones del congreso se instó a "solicitar al departamento respectivo de la UNESCO, la creación de una Escuela Panamericana de Educación Física” (Memoria oficial del VII Congreso Panamericano de Educación Física, 1980, p. 85; Calderón \& Ospina 1981).

Cabe mencionar que, a pesar de los incipientes movimientos latinoamericanos vinculados con la segunda ola feminista (Carosio, 2012; Sagot, 2017) y la importancia asignada en las agendas educativas latinoamericanas a la educación física de niñas, adolescentes y mujeres adultas, la participación de las mujeres en el evento internacional fue mucho menor con relación a la presencia masculina. La mayoría de los momentos de mayor visibilidad, prestigio y autoridad en el evento fueron ocupados por varones. Por ejemplo, en la apertura y la clausura del congreso hubo voces masculinas. Asimismo, de las cinco ponencias centrales, solo una fue pronunciada por una mujer. El Comité organizador nacional tuvo solo presencia masculina. De los catorce vicepresidentes electos de diferentes países, solo tres fueron mujeres: Sonia Toro Seda, por Puerto Rico; Martha Moncada de Rojas, por Colombia; y María Josefa Lora, por Perú (Memoria oficial del VII Congreso Panamericano de Educación Física, 1980, p. 5). El estratégico Comité Permanente del Congreso no tuvo una mujer hasta el X Congreso Panamericano de Educación Física realizado en Cuba, La Habana en 1986 (Memorias del X Congreso Panamericano de Educación Física, 1987, pp. 58-142). Del total de inscriptos en el congreso, únicamente el 15\%, aproximadamente, correspondió a mujeres (Memoria oficial del VII Congreso Panamericano de Educación Física, 1980, pp. 98-103).

A principios de los ochenta, los discursos y las prácticas vinculadas a la educación y al deporte expuestas en el congreso, salvo excepciones, continuaron naturalizando determinados deportes, algunas actividades lúdicas y ciertas gimnasias solo para niños o solo para niñas, esencializando el desarrollo de cualidades corporales y finalidades diferentes de acuerdo al 'sexo' y jerarquizando de forma estereotipada e injusta el acceso a ciertos espacios, materiales, vestimentas y competencias deportivas. En síntesis, los expertos y el gobierno de las instituciones vinculadas con el universo de la educación física y el deporte siguieron predominantemente en manos de ciertos varones y su mirada, salvo excepciones, siguió siendo androcéntrica.

\section{Conclusiones}

El Séptimo Congreso Panamericano de Educación Física realizado en Santo Domingo a principios de los años ochenta consolidó un espacio de sociabilidad e intercambio entre agentes de diferentes países e instituciones, que tenían una historia previa de casi cuatro décadas. Los temas abordados fueron diversos, aunque ciertos enfoques prevalecieron en las presentaciones; entre ellos, la preocupación por el planeamiento educativo traducido en un diseño curricular racional e integrado para la Educación Física -detallando los objetivos mensurables, las actividades, la formación de recursos humanos y las finalidades-. Muchas de las exposiciones estuvieron atravesadas por una concepción tecnocrática de la educación, basada en una tradicional pedagogía por objetivos, que priorizó más el cómo de la educación física que el por qué y el para qué, en el marco de procesos más generales de políticas educativas vinculadas con la modernización a través del 'planeamiento educativo', con el fin de mejorar y optimizar el funcionamiento de los sistemas escolares nacionales.

Entre los desacuerdos y los conflictos producidos en el congreso aparecieron las ya comunes críticas a la tradición autoritaria en la enseńanza de la disciplina, 
la urgencia por mejorar la formación profesional y, sobre todo, la necesidad de que se eviten las posibles influencias deformantes del deporte de alto nivel competitivo en las clases de educación física.

Junto con la circulación de saberes, enfoques y sentidos sobre la Educación Física, los deportes o el currículum, el evento consolidó a un grupo de expertos en el arte de educar y curar a través del movimiento. Amparados y legitimados a partir de estructuras estatales -cada vez más sólidas y con mayores recursos-, como las Direcciones Generales de Educación Física o de Deportes, los Ministerios o Secretarías de Higiene o Salud, las instituciones de formación docente, como las terciarias o universitarias o las instituciones militares, un pequeño grupo de personas se convirtió en el referente legítimo a la hora de producir, transmitir y distribuir sentidos específicos sobre la Educación Física, los deportes, la pedagogía, el currículum y sus diferentes finalidades. Un nuevo grupo de expertos desplazó y reemplazó a los referentes que habían construido el espacio entre los años cuarenta y los años setenta. Entre los 'nuevos' referentes se destacó la figura de Carlos Vera Guardia, acompañado y apoyado por un importante grupo de profesores y especialistas en la materia.

Pero, el congreso de Santo Domingo consolidó y amplificó a partir de 1980 un proceso que ya se estaba configurando, aunque muy lentamente. Estuvo relacionado con la cada vez mayor participación que tuvieron expertos y representantes de instituciones y organismos internacionales vinculados a la especialidad. Las redes y alianzas transnacionales incrementaron notablemente sus acciones en los años ochenta y noventa, obteniendo no solo legitimidad en las funciones e intervenciones fuera y dentro del Estado, sino logrando recursos económicos y legitimación política. Únicamente en la década de los ochenta se realizaron seis congresos panamericanos (República Dominicana, 1980; México, 1982; Curazao, 1984; Cuba, 1986; Venezuela, 1987 y Guatemala, 1989); y en los noventa cinco congresos (Colombia, 1991; Costa Rica, 1993; Perú, 1995; Ecuador, 1997 y Panamá, 1999), la mayoría de ellos multitudinarios y con un importante apoyo estatal y de agencias internacionales.

\section{Referencias}

Acta final del VI Congreso Panamericano de Educación Física y II Gimnasiada Americana, Tomo I. (1976). Universidad de Carabobo.

Arata, N. \& Pineau, P. (coord.) (2019). Latinoamérica: la educación y su historia. Nuevos enfoques para su debate y enseñanza. Editorial de la Facultad de Filosofía y Letras, Universidad de Buenos Aires.

Arnove, R. (2006). La educación en América Latina: Dependencia, subdesarrollo y desigualdad. En Bonal, X. (ed.), Globalización, educación y pobreza en América Latina: Hacia una nueva agenda politica (47-68). Editorial Bellaterra.

Butler, J. (2019). Cuerpos aliados y lucha politica. Hacia una teoría performativa de la asamblea. Paidós.

Calderón, A. \& Ospina J. A. (1981). Conclusiones generales del VII Congreso Panamericano de Educación Física. Educación Física y Deporte, 3(2), 73-77. https://revistas.udea.edu. co/index.php/educacionfisicaydeporte/article/ view/22866

Carosio, A. (Coord.) (2012). Feminismo y cambio social en América Latina y el Caribe. CLACSO.

Díaz Barriga, A. (1996). El currículo escolar. Surgimiento y perspectiva. Aique.

Documentos de Trabajo, Memorias del IV Congreso Panamericano de Educación Física. (1965). s/e.

Eusse, K. L. G., Almeida, F. Q. \& Bracht, V. (2019). ¿Esportivização? da educação física colombiana: a "herança" do convênio Colombo Alemão nas páginas da revista Educación Física y Deporte. Revista Brasileira de Ciencias do Esporte, 41(4), 437-443. https://doi.org/10.1016/j.rbce.2018. 08.002

Furment, O. (21 de noviembre de 1980). Finaliza hoy Congreso de Educación Física. Listín Diario.

Galak, E. L. (2019). Fortalecer los músculos para mejorar la raza. Discursos eugenésicos sobre educación del cuerpo en revistas pedagógicas de Argentina y Brasil (1920-1940). Sociohistórica, 44, e086. https://doi.org/10.24215/1852 $1606 \mathrm{e} 086$ 
Gleyse, J. \& Scharagrodsky, P. (2013/2). Le Dr. Enrique Romero Brest, ses visites aux insitutions européennes de formation et le Congrès d'Education Physique de Paris en 1913 comme indicateurs de la mondialisation et de la nationalisation de la 'culture physique'. Revue Staps, 100, 89-107. https://www.cairn.info/revue-staps-2013-2page-89.htm

Goodson, I. (2003). Estudio del currículum. Casos y métodos. Amorrortu Editores.

Informe del III Congreso Panamericano de Educación Física. (1950). Comisión Nacional de Educación Física (CNEF).

Kirk, D. (2010). Physical Education Futures. Routledge. Levoratti, A. (2020). La configuración de la Dirección Nacional de Educación Física, Deportes y Recreación de la República Argentina. Entre las problemáticas locales y los lineamientos internacionales (1963-1983). Cuaderno Venezolano de Sociología, 29(2), 110-129. https://dialnet. unirioja.es/servlet/revista?codigo $=12537$

Linhales, M., Rodrigues Puchta \& Rosa, M. (org.) (2019). Diálogos Transnacionais na História da Educação Física. Fino Traço.

Melo, V. \& Fortes, R. (2010). História do esporte: panorama e perspectivas. Fronteiras, 12(22), 11-35.

Memoria del V Congreso Panamericano de Educación Física. Desarrollo y relatos. (1971). Administración de Educación Física, Deportes y Recreación. Ministerio de Cultura y Educación. Talleres Gráficos Yunke.

Memoria oficial del II Congreso Panamericano de Educación Física. (1946). Dirección Nacional de Educación Física y Enseñanza Premilitar.

Memoria oficial del VII Congreso Panamericano de Educación Física. (1980). Secretaría de Estado, Educación, Bellas Artes y Cultos.

Memorias del X Congreso Panamericano de Educación Física. (1987). Unidad Impresora 'José A. Huelga', Instituto Nacional de Deportes, Educación Física y Recreación (INDER).

Moncada de Rojas, M. (1981). La Educación Física en América en la década de los 80. Educación Física
Y Deporte, 3(1), 7-12. https://revistas.udea.edu. co/index.php/educacionfisicaydeporte/article/ view/22806

O que foi o I congresso panamericano de educação física. (1944). Revista Brasileira de Educação Fisica, I(1), 18-32.

Quitzau, E.M. \& Moraes e Silva, M. (2020). Educación física y ciencia: una mirada historiográfica. Revista de la Asociación Latinoamericana de Estudios Socioculturales del Deporte, 12(1), 23-42.

Rodríguez, R. (15 de noviembre de 1980). Guzmán presidirá la apertura del Congreso de Educación Física. El Caribe.

Rodríguez, R. (17 de noviembre de 1980). Abren VII Congreso de Educación Física. El Caribe.

Rodríguez, R. (18 de noviembre de 1980). Congreso analiza situación de Educación Física. El Caribe.

Rodríguez, R. (19 de noviembre de 1980). Delegados Congreso Educación destacan la importancia del cónclave. El Caribe.

Rodríguez, R. (20 de noviembre de 1980). Aplicarán RD resultados Congreso Educación Física; Califica de interesantes ponencias presentadas en el evento. El Caribe.

Rodríguez, R. (21 de noviembre de 1980). Cree confusión Educación Física con Deporte impide su desarrollo. El Caribe.

Rodríguez, R. (22 de noviembre de 1980). Clausuran VII Congreso de Educación Física. El Caribe.

Sagot, M. (coord.) (2017). Feminismos, pensamiento crítico y propuestas alternativas en América Latina. CLACSO.

Saraví Rivière, J. (2014). Historia de la Educación Física argentina. Editorial del Zorzal.

Scharagrodsky, P. (2014). Palabras preliminares. En Scharagrodsky, P. (comp.). Miradas médicas sobre la cultura fisica en Argentina (pp. 9-12). Prometeo.

Scharagrodsky, P. (2020). Especialistas, instituciones y propuestas vinculadas al saber y al hacer corporal: la Primera Conferencia de Profesores de Educación Física, Buenos Aires, 1942. Resgate: Revista Interdisciplinar de Cultura, 28, e020028. 
Scharagrodsky, P. (comp.) (2011). La invención del homo gymnasticus. Fragmentos históricos sobre la educación de los cuerpos en movimiento en occidente. Prometeo.

Serrano, F.V. (2010). Estado, golpes de Estado y militarización en América Latina: una reflexión histórico política. Argumentos, 23(64), 175-193. http:// www.scielo.org. $\mathrm{mx} / \mathrm{scielo}$.php?script=sci arttext\&pid=S0187-57952010000300008

Silva, T. T. (2001). Espacios de Identidad. Una Introducción a las teorías del curriculum. Octaedro.
Suasnábar, C. (2017). Los ciclos de reforma educativa en América Latina: 1960, 1990 y 2000. Revista Española de Educación Comparada, 30, 112-135. https://doi.org/10.5944/reec.30.2017.19872

Torres, C. \& Scharagrodsky, P. (Ed.) (2019). El rostro cambiante del deporte. Perspectivas historiográficas angloparlantes (1970-2010). Prometeo. 